\title{
Recurring Candida albicans Esophagitis in a HIV-Infected Patient Undergoing Long-Term Antiretroviral Therapy, and with Absent-Negligible Immunodeficiency
}

\author{
Roberto Manfredi, Sergio Sabbatani and Leonardo Calza \\ Department of Clinical and Experimental Medicine, Division of Infectious Diseases, "Alma Mater Studiorum" University of Bologna, \\ S. Orsola-Malpighi Hospital, Bologna, Italy
}

\begin{abstract}
A patient with HIV infection developed the first episode of AIDS-defining opportunism (severe Candida albicans esophagitis) with an underlying CD4+ lymphocyte count of 1,025 cells/ $\mu \mathrm{L}$. After treatment with a highly active antiretroviral therapy (HAART), taken with insufficient compliance and leaving a residual viral load, our patient suffered from two relapses of esophageal candidiasis, which occurred three months and seven years later, when his CD4+ lymphocyte count was 930 and 439 cells $/ \mu \mathrm{L}$, respectively, and a viral load slightly above $10^{4}$ copies $/ \mathrm{mL}$ was still present. Also in the HAART era, Candida esophagitis remains one of the most common AIDS-defining diseases, but a presentation with a concurrent $\mathrm{CD4}+$ count above 1,000 cells $/ \mu \mathrm{L}$ remains a rare exception, as well as the two isolated, subsequent relapses, occurred with a CD4+ count ranging from 439 to 930 cells/ $\mu \mathrm{L}$, and a residual HIV viremia due to insufficient adherence to the prescribed HAART regimens. Our case report represents the opportunity to revisit the epidemiology and, especially, the pathogenesis of this opportunistic fungal complication in HIVinfected patients and in other subjects at risk, on the ground of an extensive literature review, and to explore possible alternative supporting factors other than the crude absolute CD4+ lymphocyte count, with emphasis on the possible role of a persisting HIV viremia, and other potential contributing factors. Clinicians engaged with immunocompromised patients and subjects with HIV disease, should be aware that a Candida esophagitis may occur and relapse also when the cell-mediated immunity, as measured by a simple CD4+ cell count, do not show relevant abnormalities.
\end{abstract}

Key-Words: Esophageal candidiasis, HIV infection, CD4+ lymphocyte count, HIV viral load, recurring opportunistic disease, antiretroviral therapy, negligible or absent immunodeficiency.

In the era of highly active antiretroviral therapy (HAART) a dramatic drop of immunodeficiency-related opportunism occurred (especially for disorders associated with a CD4+ lymphocyte count below 50-100 cells/ $\mu \mathrm{L}$, like cryptosporidiosis, cytomegalovirosis, atypical mycobacteriosis, isosporiasis, neurotoxoplasmosis, and cryptococcosis) [1-5], compared with the years preceding the introduction of potent antiretroviral combinations (before mid1996) [6-8]. However, in the HAART era the distribution of the main AIDS-defining diseases remained somewhat unchanged in their respective proportion [1-4, 9]. Also recent experiences proved that opportunistic infections are still the major causes of morbidity and mortality in the HAART era $[1,3,4,10-12]$ and the same epidemiological pattern has been very recently confirmed among HIV-infected children [13].

As a consequence, esophageal candidiasis and Pneumocystis carinii pneumonia remained the most frequent AIDS-defining disorders, a decade after the introduction of HAART (1996-2006).

In fact, the profile of AIDS notifications in Italy updated on December 31, 2005, reported 56,016 cumulative cases of AIDS, with only 1,577 new cases registered in the last year,

Received on 20 August 2007; revised 15 October 2007.

Address for correspondence: Dr. Roberto Manfredi. Associate Professor of Infectious Diseases, University of Bologna. Infectious Diseases, S. Orsola Hospital. Via Massarenti 11 - I-40138 Bologna, Italy. Telephone: +39-051-6363355. Telefax: +39-051-343500. E-mail: Roberto.manfredi@unibo.it.

The Brazilian Journal of Infectious Diseases 2007;11(6):605-609. (C) 2007 by The Brazilian Journal of Infectious Diseases and Contexto Publishing. All rights reserved.
2005 [10]. According to these data, esophageal and pulmonary candidiasis and pneumocystosis remain the leading causes of full-blown AIDS, accounting for nearly $40 \%$ of overall cases, although a slight decrease of visceral candidiasis was observed in newly diagnosed AIDS patients (both adults and children), who were already taking HAART (from $25.3 \%$ of all AIDSrelated disorders observed in the years preceding 1995, down to $18.7 \%$ in the years 2004-2005) [10]. This last issue could reflect a more efficient immune response to fungal pathogens in patients recovering immune defence when using HAART, but could indirectly confirm the direct antifungal activity of protease inhibitors, already demonstrated in elegant ex vivo studies [14], and apparently confirmed by preliminary epidemiological and clinical observations [15]. However, among HAART-treated novel AIDS patients, visceral candidiasis still ranks first among AIDS-defining diseases (23.7\% of cases) [10]. When assessed according to HIV plasma viral load and CD4+ lymphocyte count, the frequency of esophageal candidiasis was surprisingly greater among patients with an undetectable or a very low HIV viremia (below 500 copies $/ \mathrm{mL}$ ), but a CD4+ count above 200 cells $/ \mu \mathrm{L}$ at diagnosis (17.6\%), compared with those with $<200$ cells/ $\mu \mathrm{L}(16.3 \%$ ), while among viremic patients (viral load $>500$ copies/mL), a CD4+ count $<200$ cells $/ \mu \mathrm{L}$ prompted a visceral candidiasis in more subjects than in those with a CD4+ lymphocyte count above 200 cells $/ \mu \mathrm{L}$ (26.3\% versus $23.9 \%$ ) [10].

An atypical case report of Candida albicans esophagitis prompting a diagnosis of AIDS in presence of a CD4+ lymphocyte count exceeding 1,000 cells/ $\mu \mathrm{L}$, with two subsequent relapses occurring three months and seven year 
later, when a HAART therapy was conducted with incomplete patient's adherence, although ensuring a CD4+ count of 930 and 439 cells $/ \mu$ L respectively, while HIV viremia remained slightly over $10^{4}$ copies $/ \mathrm{mL}$, is described and discussed in its epidemiological, clinical, and especially pathogenic implications, on the basis of an extensive and updated literature review.

\section{Case Report}

A 32-year-old homosexual man followed for 11 years for a $\mathrm{HIV} / \mathrm{HCV}$ co-infection started seven years ago his first antiretroviral therapy with a didanosine-stavudine-indinavir combination (since October 1999); dysphagia and epigastralgia appeared and progressively worsened, and a mild, associated oropharyngeal candidiasis became apparent. Upon hospitalization, his CD4+ count was 1,025 cells $/ \mu \mathrm{L}$ (CD4+ percentage $44 \%$, leading to a CD4+/CD8+ rate $>1$ ), while 43,800 copies/mL of HIV RNA were detected. An esophagogastroduodenoscopy showed an extensive esophageal candidiasis, associated with a mild hiatal hernia. The histopathologic examination of biopsy specimens showed a chronic and abundant inflammatory infiltrate involving the lamina propria, mostly represented by mononuclear cells and macrophages, with a prominent amount of Candida blastosporae and pseudohyphae. Upon microbiological assays, a Candida albicans strain which resulted susceptible in vitro to all available antifungal agents was repeatedly isolated. An antimycotic course was carried out with oral itraconazole ( $400 \mathrm{mg}$ /day for the first week, than $200 \mathrm{mg}$ daily for two more weeks, plus adequate supportive therapy, and a further endoscopic examination carried out one month after the first one confirmed the complete disappearance of clinical signs of Candida esophagitis, registered in the meantime by our patient. During the subsequent months, the immunological and virological response to HAART was effective (viral suppression achieved after one month only, branched DNA assay), and patient's compliance and tolerability were favorable. An antifungal chemoprophylaxis was not administered due to the very elevated CD4+ cell count.

Unexpectedly, three months later (January 2000), when the patient's CD4+ count accounted for 930 cells $/ \mu \mathrm{L}$, a novel episode of endoscopy-confirmed esophageal candidiasis occurred, and it was treated with a two-week course of oral fluconazole (400 mg/day). However, viral load became detectable (10,200 HIV-RNA copies/mL), and our patient complained of a low adherence to indinavir thrice daily, so that the HAART regimen was changed to comply with our patient's requirements. During the subsequent years, antiretroviral treatment was modified many times (mainly due to repeated gastrointestinal intolerance and insufficient patient's adherence levels), until a regimen containing lamivudine, tenofovir, and efavirenz, conducted since October 2004. It had a favorable immunological response (a CD4+ lymphocyte count persistently above 500 cells $/ \mu \mathrm{L}$ ), and an incomplete viral suppression (viremia slightly above $10^{4}$ copies $/ \mathrm{mL}$ ). Neither opportunistic infections nor clinical events of any type (including esophagitis and candidiasis) occurred in the meantime, as well as relevant intolerance to undergoing HAART. No abnormalities were found when investigating chemotaxis, killing, oxidative burst and phagocytosis of peripheral polymorphonuclear cells, as well as in vitro immunoglobulin synthesis and blastization of B- and Tlymphocytes.

Finally, nine months ago (December 2005), the initial reappearance of a mild oropharyngeal candidiasis followed by signs and symptoms of an esophagitis prompted another esophagogastroduodenoscopy, which was performed in early February 2006, when our patient suffered again from a prominent dysphagia and had an evident oropharyngeal candidiasis (despite topical therapy attempts with nistatine and amphotericin B troches), and spontaneously interrupted the ongoing antiretroviral therapy. The endoscopy examination detected another severe recurrence of Candida albicans esophagitis, as confirmed by combined mycological and histopathological studies. In vitro sensitivity studies of available antimycotic compounds did not show any change compared with previous results. At that time, the CD4+ count of our patient was 439 cells/ $\mu \mathrm{L}$ (CD4+ percentage $29 \%$ ), while HIV RNA copies rose to $42,400 / \mathrm{mL}$. After a novel treatment course with i.v. fluconazole ( $600 \mathrm{mg} /$ day for a week), followed by oral fluconazole (400 mg/day for two weeks), a complete resolution of all signs and symptoms of esophageal candidiasis was achieved, and antiretroviral therapy was safely resumed.

\section{Discussion}

The esophagus infections are unusual in the general population and strongly imply some grade of immunodeficiency or underlying disease, although apparently immunocompetent individuals may not be totally exempt. For all the immunocompromised patients, the most frequently identified esophageal pathogens are Candida spp., cytomegalovirus, and herpes simplex virus [16], although more rare etiologies (i.e. a bacterial one) [17] can not be excluded. Accurate and timely diagnoses are mandatory, as effective treatments are available for almost all microbial etiologies. On the other hand, the retrieval of esophageal signs and symptoms are not necessarily due to an infectious process, and possible alternative diagnoses, like acid-peptic injury, drug-induced esophagitis, or structural and functional abnormalities should not be overlooked when a state of immunodeficiency (including HIV disease) is of concern [7,16]. Inhaled corticosteroids taken for a long time may also prompt esophageal candidiasis, usually preceded by an oral Candida localization [18].

Until the date of the end of this study, a low absolute CD4+ lymphocyte count remains a major, independent risk factor for the development of major opportunistic infections (including visceral-disseminated candidiasis), while a preexisting oropharyngeal candidiasis is the more obvious source of an esophageal extension of disease $[1,11,19,20]$, although 
this last issue is not always retrieved in all patients suffering from esophageal candidiasis [8]. In fact, a number of cohort studies, either prospective or retrospective in nature, confirmed the elevated incidence of visceral candidiasis among immunocompromised HIV-infected patients, and its strict relationship with the peripheral CD4+ lymphocyte count, with a threshold usually placed at 200 cells/ $\mu \mathrm{L}[1,3,4,11]$. Among a very elevated number of literature contribution, Abgrall et al. [20] assessed risk factors for a first episode of esophageal candidiasis in a cohort study including 1,368 French patients treated before the HAART era: amongst the 87 patients $(6 \%)$ who developed a Candida esophagitis in a median follow-up time of 19 months, a low baseline CD4+ count $(\mathrm{p}<.0001)$ was the most important predictive factor, followed by a high baseline viremia $(\mathrm{p}<.0001)$, a recent antibacterial chemotherapy $(\mathrm{p}=.01)$, an oral candidiasis $(\mathrm{p}<.05)$, and a cotrimoxazole prophylaxis $(\mathrm{p}=.04)$ [20]. In another large clinical cohort, only a few cases of esophageal candidiasis, $P$. carinii pneumonia, and toxoplasmic encephalitis were reported with a CD4+ lymphocyte count exceeding 300 cells/ $\mu \mathrm{L}$ [4].

However, probable pathogenic pathways which are somewhat independent from the crude, peripheral CD4+ lymphocyte count should be advocated to explain our exceptional case of confirmed esophageal candidiasis with a CD4+ count of 1,025 cells/ $\mu \mathrm{L}$, subsequently relapsed despite complete clinical and microbiological cure of previous episodes, immediate HAART introduction, no other evident predisposing factors, and a level of CD4+ lymphocytes of 930 and 439 cells $/ \mu \mathrm{L}$, in the two subsequent recurrences, respectively.

In HAART-treated patients, a partial, function-limited immune reconstitution syndrome has been advocated as a cause supporting relapses of cytomegalovirus retinitis occurring when the absolute CD4+ cell count overcome 300 cells/ $\mu \mathrm{L}[21]$. Immunological assays investigating the status of the immune response against specific pathogens probably warrant further investigation in the setting of HIV disease and other immunodeficiency disorders. The progression and extent of Candida colonization in patients at risk for visceral candidiasis also deserve high attention, but the hypothesis to use nonabsorbable antifungal drugs seems effective only in avoiding candidemia and focal localizations in haematological and surgical patients, and transplant recipients [22].

When focusing on HIV diseases, some concomitant infections, either opportunistic of not, have been anecdotally claimed as possible co-factors of a decreased immune response and increased HIV viral load, possibly leading to adjunctive infectious complications [23-25]. It is the case of pulmonary and extrapulmonary tuberculosis [24], but also of newly diagnosed syphilis [23]. An increased or a persistently elevated HIV viral load has been claimed as an isolated supportive factor of the development or the relapse of opportunistic infections, even when no significant changes and consequences were observed on CD4+ lymphocyte count
[24], as previously observed [5,10,26]. Moreover, just opportunistic infections have been postulated to determine a transient increase of HIV viremia, through exposure of the immune system network to microbial antigens, even in chronically infected patients undergoing HAART [25,26]. In a very interesting investigation of the pathophysiology of Candida spp. gastrointestinal colonization and disease performed in $83 \mathrm{HIV}$-infected individuals, Gottfredsson et al. [26] found a strong relationship with HIV viral load $(p=0.001)$, while surprisingly it was not the same for CD4+ lymphocyte count ( $p=0.36$ ); a multivariate assessment established that plasma viral load was the only parameter which correlated with the extent of candida colonization $(p=0.003)$ [26]. In the same year (1999) another study [5] assigned a prognostic value to the viral load amount in the natural history of HIVassociated pneumocystosis, cytomegalovirosis, and atypical mycobacteriosis, in a multicentre cohort of 734 antiretroviralnaïve patients, independently of the CD4+ cell count [5]. More recently, an interim analysis of an Italian cohort of 3,023 [27], showed a higher rate of clinical progression for people with a current viral load above $10^{4}$ copies $/ \mathrm{mL}$, with a significantly increased risk of progression related to the extent and the duration of elevated HIV viremia, while a controlled (undetectable) HIV viremia seemed to confer protection against clinical progression [27]. Although it is necessary to remind that both CD4+ lymphocyte count and viral load have been usually identified as very strong predictors of morbidity and mortality in the setting of HIV disease, as the survey of Seage et al. showed in its data set of 2,193 patients [28], the persisting, low-level viremia of our patient might have played some role in prompting a recurrent esophageal candidiasis, even with an elevated CD4+ lymphocyte count.

On the other hand, in patients without HIV infection, Candida esophagitis remains an infrequent occurrence prompted by primary or secondary (iatrogenic) immunosuppression, immunologic disorders, underlying malignancies and their treatment, diabetes mellitus, and extensive surgery (i.e. gastrectomy), although some chronic medical treatments (like H2-blockers) were included into conditions possibly supporting Candida esophagitis [7]. However, in the series of 3,501 endoscopic examinations of the upper gastrointestinal tract published by Naito and coworkers, an esophageal candidiasis was diagnosed also in seven so-defined healthy patients [7], and this event is also suggested as a rare but possible event, by Baehr and McDonald [16].

When considering diagnostic issues in clinical practice, since the first years of HAART availability it has been repeatedly underlined that clinicians engaged in the management of HIV disease should take into careful consideration multiple AIDS-associated disorders [29-31], which are expected to involve with a greater incidence the socalled "AIDS presenters", i.e. individuals who could not benefit from antiretroviral therapy since their HIV seropositivity has been missed or neglected for a long time, 
and have a very severe immunodeficiency at presentation. Also atypical presentations of opportunism, probably due to the so-called immune restoration syndrome, or a different time and extent of immunological and virological response to antiretroviral therapy $[32,33]$ should be taken into careful attention. The involvement of the esophagus can not be necessarily preceded by an evident oropharyngeal localization of candidiasis [8], but it was not the case of our patient, who suffered from a mild oropharyngeal candidiasis before and at the time of diagnosis of his Candida esophagitis.

From a therapeutic point of view, the selection of potentially effective systemic antifungal agents recently broadened (with the inclusion of liposomal amphotericin B formulations, caspofungin, voriconazole, and other more recent compounds), although the majority of Candida albicans strains are expected to be responsive to fluconazole and itraconazole [8,34] (as in the present case), and usually treatment with other agents is not required [35]. In the light of the HAART effectiveness in prompting rapid and remarkable immune recovery, a prolonged systemic antifungal prophylaxis is no more considered in the HAART era, due to an unfavorable cost-effectiveness rate, and especially the risk to induce and spread azole resistance, and to select azole-unresponsive Candida strains, when administering low-dose, long-term oral antifungal agents [36-38].

In conclusion, the occurrence of an AIDS diagnosis in a patient who developed a confirmed, antifungal-susceptible Candida albicans esophagitis when his CD4+ lymphocyte count was 1,025 cells $/ \mu \mathrm{L}$, and suffered from two relapses of esophageal candidiasis despite HAART introduction, and in presence of a favorable CD4+ count $(930$ and 439 cells $/ \mu \mathrm{L}$, respectively), invites to consider other potential co-factors supporting a so rare occurrence. In our case, a repeatedly present viral replication (slightly over $10^{4} \mathrm{HIV}$-RNA copies/ $\mathrm{mL}$ ), due to an insufficient compliance with HAART, has been considered and discussed, but the persisting phenomenon of opportunism in the HAART era deserves further investigation from an epidemiological, pathogenic, and clinical point of view.

\section{References}

1. Mocroft A., Youle M., Phillips A.N., et al. The incidence of AIDSdefining illnesses in 4883 patients with human immunodeficiency virus infection. Royal Free/Chelsea and Westminster Hospitals Collaborative Group. Arch Intern Med 1998;158:491-7.

2. Manfredi R. Effects of the introduction of highly active antiretroviral therapy (HAART) on the temporal trend of diagnosis of AIDS and AIDS-defining disorders. Infez Med 2000;8:134-8.

3. Kaplan J.E., Hanson D., Dworkin M.S., et al. Epidemiology of human immunodeficiency virus-associated opportunistic infections in the United States in the era of highly active antiretroviral therapy. Clin Infect Dis 2000;30(Suppl. 1):S5-14.

4. Yazdanpanah T., Chene G., Losina E., et al. Incidence of primary opportunistic infections in two human immunodeficiency virus-infected French clinical cohorts. Int J Epidemiol 2001;30:664-71.
5. Lyles R.H., Chu C., Mellors J.W., et al. Prognostic value of plasma HIV RNA in the natural history of Pneumocystis carinii pneumonia, cytomegalovirus and Mycobacterium avium complex. Multicenter AIDS Cohort Study. AIDS 1999; $13: 341-9$.

6. Manfredi R., Nanetti A., Mazzoni A., et al. The incidence, etiology, and clinical significance of visceral mycoses in patients with AIDS. Minerva Med 1993;84:383-91.

7. Naito Y., Yoshikawa T., Oyamada H., et al. Esophageal candidiasis. Gastroenterol Jpn 1988;23:363-70.

8. Knobber D., Brusis T. Clinical aspects of therapy of esophageal candidiasis. Laryngol Rhinol Otol (Stuttg) 1985;64:314-7.

9. Manfredi R., Calza L., Chiodo F. Lack of change in the distribution of AIDS-defining opportunistic diseases and the related degree of immunodeficiency during the periods before and after the introduction of highly active antiretroviral therapy. Eur J Clin Microbiol Infect Dis 2001;20:410-3.

10. Suligoi B., Boros S., Camoni L., et al. Aggiornamento dei casi di AIDS notificati in Italia e delle nuove diagnosi di infezione da HIV. Centro Operativo AIDS, Istituto Superiore di Sanità, Rome, issued on December 2005:1-24.

11. Jing W., Ismail R. Mucocutaneous manifestations of HIV infection: a retrospective analysis of 145 cases in a Chinese population in Malaysia. Int J Dermatol 1999;38:457-63.

12. Smit C., Geskus R., Walker S., et al. CASCADE Collaboration. Effective therapy has altered the spectrum of cause-specific mortality following HIV seroconversion. AIDS 2006; $20: 741-9$.

13. Gona P., Van Dyke R.B., Williams P.L., et al. Incidence of opportunistic and other infections in HIV-infected children in the HAART era. JAMA 2006;296:292-300.

14. Cassone A., Tacconelli E., De Bernardis F., et al Antiretroviral therapy with protease inhibitors has an early immune reconstitution-independent beneficial effect on Candida virulence and oral candidiasis in human immunodeficiency virusinfected subjects. J Infect Dis 2002;185:188-95.

15. Manfredi R., Calza L., Chiodo F. AIDS-defining fungal opportunism in the HAART era. Trend of frequency and reduced incidence when HIV protease inhibitors are administered. Clin Microbiol Infect 2006;12(Suppl. 4): 1226.

16. Baehr P.H., McDonald G.B. Esophageal infections: risk factors, presentation, diagnosis, and treatment. Gastroenterology 1994; 106:509-32.

17. Mastroianni A., Cancellieri C., Coronado O., et al. Bacterial esophagitis in patients with HIV disease. Infez Med 1999; 7:192-4.

18. Simon M.R., Houser W.L., Smith K.A., Long P.M. Esophageal candidiasis as a complication of inhaled corticosteroids. Ann Allergy Asthma Immunol 1997;79:333-8.

19. Gupta E., Vajpayee M., Xess I., Seth P. Oral candididasis in HIVinfected patients and its relation to CD4+ counts: an adjunct clinical marker of HIV disease progression. Trop Doct 2006;36: 127 .

20. Abgrall S., Charreau I., Joly V., et al. Delta Coordinating Committee. Risk factors for esophageal candidiasis in a large cohort of HIVinfected patients treated with nucleoside analogues. Eur J Clin Microbiol Infect Dis 2001;20:346-9.

21. Lilleri D., Piccinini G., Baldanti F., et al. Multiple relapses of human cytomegalovirus retinitis during HAART in an AIDS patient with reconstitution of $\mathrm{CD} 4+\mathrm{T}$ cell count in the absence of HCMV-specific CD4+ T cell response. J Clin Virol 2003;26:95-100.

22. Bonavina L., Incarbone R., Reitano M., et al. Candida colonization in patients with esophageal disease: a prospective clinical study. Dis Esophagus 2003; 16:70-2.

23. Buchacz K., Patel P., Taylor M., et al. Syphilis increases HIV viral load and decreases CD4 cell counts in HIV-infected patients with new syphilis infections. AIDS 2004;18:2075-9. 
24. Day J.H., Grant A.D., Fielding K.L.,et al Does tuberculosis increase HIV load? J Infect Dis 2004;190:1677-84.

25. Jones L.E., Perelson A.S. Opportunistic infection as a cause of transient viremia in chronically infected HIV patients under treatment with HAART. Bull Math Biol $2005 ; 67: 1227-51$

26. Gottfredsson M., Cox G.M., Indridason O.S., et al. Association of plasma levels of human immunodeficiency virus type 1 RNA and oropharyngeal Candida colonization. J Infect Dis 1999; 180:534-7.

27. Murri R., Lepri A.C., Cicconi P., et al. ICoNA Study Group. Is moderate HIV viremia associated with a higher risk of clinical progression in HIV-infected people treated with highly active antiretroviral therapy: evidence from the Italian cohort of antiretroviral-naïve patients study. J Acquir Immune Defic Syndr 2006; $41: 23-30$

28. Seage G.R. $3^{\text {rd }}$, Losina E., Goldie S.J., et al. The relationship of preventable opportunistic infections, HIV-1 RNA, and CD4 cell counts to chronic mortality. J Acquir Immune Defic Syndr 2002;30:421-8.

29. Manfredi R., Calza L., Chiodo F. Three to seven concurrent AIDSdefining disorders at first hospitalization of AIDS presenters as an unexpected emerging feature during the era of highly active antiretroviral therapy. AIDS 2002;16:2356-8.

30. Manfredi R., Calza L., Chiodo F. Dual Candida albicans and Cryptococcus neoformans fungaemia in an AIDS presenter: a unique disease association in the highly active antiretroviral therapy (HAART) era. J Med Microbiol 2002;51:1135-7.
31. Manfredi R., Fulgaro C., Legnani G., et al. Emergence of amphotericin B.resistant Cryptococcus laurentii meningoencephalitis shortly after treatment for Cryptococcus neoformans meningitis in a patient with AIDS. AIDS Patient Care STDs 2006;20:227-32.

32. Stone S.F., Price P., French M.A. Immune restoration disease: a consequence of dysregulated immune responses after HAART. Curr HIV Res 2004;2:235-42.

33. Manfredi R., Pieri F., Pileri S.A., Chiodo F. The changing face of AIDS-related opportunism; cryptococcosis in the highly active antiretroviral therapy (HAART era. Case reports and literature review. Mycopathologia 1999;148:73-8.

34. Vazquez J.A. Therapeutic options for the management of oropharyngeal and esophageal candidiasis in HIV/AIDS patients. HIV Clin Trials 2000; $1: 47-59$.

35. Dinubile M.J., Lupinacci R.J., Berman R.S., Sable C.A. Response and relapse rates of Candida esophagitis in HIV-infected patients treated with caspofungin. AIDS Res Hum Retroviruses 2002; 18:903-8.

36. Manfredi R., Mastroianni A., Coronado O.V., Chiodo F. Fluconazole as prophylaxis against fungal infection in patients with advanced HIV infection. Arch Intern Med 1997;157:64-9.

37. Bozzette S.A. Fluconazole prophylaxis in HIV disease, revisited. Clin Infect Dis 2005;41:1481-2.

38. Goldman M., Cloud G.A., Wade K.D., et al. A randomized study of the use of fluconazole in continuous versus episodic therapy in patients with advanced HIV infection and a history of oropharyngeal candidiasis: AIDS Clinical Trials Group Study 323/ Mycoses Study Group Study 40. Clin Infect Dis 2005;41:1473-80. 